\title{
Personal Identification Using Ears Based on Statistical Features
}

\author{
Alaa Tharwat \\ Electronics and Communication, Engineering and Technology, Arab Academy for Science, \\ Technology and Maritime Transport (AASTMT), Cairo, Egypt \\ Advisor/s: Atalla I. Hashad, Gouda I. Salama \\ Date and location of PhD thesis defense: May 2014, AASTMT
}

Received 18th February 2015; accepted 27th July 2015

\begin{abstract}
Biometrics is an automated method of recognizing a person based on physiological (e.g. face, iris, or retina) or behavioral (e.g. gait, signature, or dynamic keystrokes) characteristics. Ear recognition is one of the physiological biometrics' types that have been gaining interested in the recent years. Ear recognition, achieves good accuracy and has many advantages such as it is not affected by expressions, health, and it is more stable than many other biometrics. However, it has many challenges such as the pose of the face, lighting variation, occlusion with hair or clothes.

In this research, four proposed models are used to identify people using ear images. The first model used single feature extraction method based on single classifier. While, the second model used single feature extraction method based on multi-classifiers. The third model used feature combination techniques (parallel or serial) based on single classifier. Finally, in the fourth model multi-features and multi-classifiers are used. In this research, there are four methods that are used to extract the features, namely, Principal Component Analysis (PCA), Linear Discriminant Analysis (LDA), Independent Component Analysis (ICA), and Discrete Cosine Transform (DCT). Neural networks, decision tree, and minimum distance classifiers are used to classify the unknown samples.

The occlusion problem with hair or scarves is one of the big challenges of the ear recognition systems. In this research, segmentation technique is proposed to neglect the occluded part and solve the occlusion problem. The idea of the segmentation technique is based on dividing the ear images into different parts. The occluded part/s is neglected and the rest of the parts are used to identify people based on features fusion and classifiers fusion. The segmentation technique consists of two main types, namely, uniform or non-uniform segmentation techniques. In this research, the uniform segmentation technique is used for many experiments (horizontal, vertical, and grid). All the four proposed models are applied to all ear segments to investigate the power of each model and to achieve a high accuracy.

In this research, ear database images is used. The ear dataset consists of 102 grayscale images (6 images for each of 17 subjects) in PGM format [1].
\end{abstract}

Correspondence to: <engalaatharwat@hotmail.com>

Recommended for acceptance by Jorge Bernal

DOI http://dx.doi.org/10.5565/rev/elcvia.704

ELCVIA ISSN:1577-5097

Published by Computer Vision Center / Universitat Autònoma de Barcelona, Barcelona, Spain 
The proposed models are achieved good identification rates using ear images. In the first model, the best accuracy achieved using LDA and neural network classifier. The results of the first model ranged from $64.12 \%$ to $100 \%$. In the second model, many classifiers are fused to increase the recognition rate. In this method, two methods are used, namely, Borda count and majority voting. The results of this model ranged from $94.12 \%$ to $96.08 \%$. In the third model, the features using two different methods, namely serial and parallel are combined. The results of this model prove that the serial combination is more powerful than parallel combination. Finally, in the fourth model, two features and two classifiers are fused to get one decision. The accuracy of this model is approximately the same of the third model, and it does not achieve good results because there is a diversity between different classifiers. Moreover, the proposed segmentation model achieved good results when some parts of the ear images are occluded.

\section{References}

[1] M. A. Carreira-Perpinan, Compression neural networks for feature extraction: Application to human recognition from ear images. MSc Thesis, Faculty of Informatics, Technical University of Madrid, Spain. 